\title{
Frequently Hospitalized Patients' Perceptions of Factors Contributing to High Hospital Use
}

\author{
Kevin J O'Leary, MD, MS*; Margaret M Chapman, MD²; Shandu Foster, MA'; Lyndsey O’Hara, RN'; \\ Bruce L Henschen, MD, MPH${ }^{3}$; Kenzie A Cameron, PhD, $\mathrm{MPH}^{3}$
}

\begin{abstract}
${ }^{1}$ Division of Hospital Medicine Northwestern University Feinberg School of Medicine, Chicago, Illinois; ${ }^{2}$ Division of General Internal Medicine, Massachusetts General Hospital, Boston, Massachusetts; ${ }^{3}$ Division of General Internal Medicine and Geriatrics, Northwestern University Feinberg School of Medicine, Chicago, Illinois.
\end{abstract}

BACKGROUND: A small proportion of patients accounts for a large proportion of hospitalizations.

OBJECTIVE: To obtain patients' perspectives of factors associated with the onset and continuation of high hospital use.

DESIGN: Qualitative research study where a research coordinator conducted one-on-one semi-structured interviews. A team of researchers performed inductive coding and analysis.

SETTING: A single urban academic hospital.

PARTICIPANTS: Patients with two unplanned 30-day readmissions within 12 months and one or more of the following: $\geq 1$ readmission in the last six months, a referral from a clinician, or $\geq 3$ observation visits.

RESULTS: Overall, 26 participants completed the interviews. Four main themes emerged. First, major medical problems were universal, but the onset of frequent hospital use varied. Second, participants perceived fluctuations in their course to be related to psychological, social, and economic factors. Social support was perceived as helpful and participants benefited when providing social support to others. Third, episodes of illness varied in onset and generally seemed uncontrollable and often unpredictable to the participants. Fourth, participants strongly desired to avoid hospitalization and typically sought care only after self-management failed.

CONCLUSIONS: Emergent themes pointed to factors which influence patients' onset of high hospital use, fluctuations in their illness over time, and triggers to seek care during an episode of illness. These findings enable patients' perspectives to be incorporated into the design of programs serving similar populations of frequently hospitalized patients. Journal of Hospital Medicine 2019;14:521-526. Published online first March 20, 2019. (c) 2019 Society of Hospital Medicine $\mathrm{n}$ recent years, hospitals have made considerable efforts to improve transitions of care, in part due to financial incentives from the Medicare Hospital Readmission Reduction Program (HRRP). ${ }^{1}$ Initially focusing on three medical conditions, the HRRP has been associated with significant reductions in readmission rates. ${ }^{2}$ Importantly, a small proportion of patients accounts for a very large proportion of hospital readmissions and hospital use.,4 Frequently hospitalized patients often have multiple chronic conditions and unique needs which may not be met by conventional approaches to healthcare delivery, including those influenced by the HRRP.4-6 In light of this challenge, some hospitals have developed programs specifically focused on frequently hospitalized patients. A recent systematic review of these programs found relatively few studies of high quality, providing only limited insight in designing interventions

*Corresponding Author: Kevin J. O'Leary MD, MS; E-mail: keoleary@nm.org; Telephone: 312-926-5924; Twitter: @kevinjolearymd

Find Additional Supporting Information in the online version of this article.

Received: October 30, 2019; Revised: January 22, 2019;

Accepted: January 27, 2019

@2019 Society of Hospital Medicine DOI 10.12788/jhm.3175 to support this population. ${ }^{7}$ Moreover, no studies appear to have incorporated the patients' perspectives into the design or adaptation of the model. Members of our research team developed and implemented the Complex High Admission Management Program (CHAMP) in January 2016 to address the needs of frequently hospitalized patients in our hospital. To enhance CHAMP and inform the design of programs serving similar populations in other health systems, we sought to identify factors associated with the onset and continuation of high hospital use. Our research question was, from the patients' perspective, what factors contribute to patients' becoming and continuing to be high users of hospital care.

\section{METHODS}

\section{Setting, Study Design, and Participants}

This qualitative study took place at Northwestern Memorial Hospital (NMH), an 894-bed urban academic hospital located in Chicago, Illinois. Between December 2016 and September 2017, we recruited adult patients admitted to the general medicine services. Eligible participants were identified with the assistance of a daily Northwestern Medicine Electronic Data Warehouse (EDW) search and included patients with two unplanned 30-day inpatient readmissions to $\mathrm{NMH}$ within the 
TABLE 1. Participant Characteristics

\begin{tabular}{|c|c|c|c|}
\hline Characteristic & $\begin{array}{l}\text { Patients without Sickle Cell Disease } \\
\qquad(\mathrm{n}=16)\end{array}$ & $\begin{array}{l}\text { Patients with Sickle Cell Disease } \\
\qquad(\mathrm{n}=10)\end{array}$ & $\begin{array}{l}\text { Total } \\
(\mathrm{N}=26)\end{array}$ \\
\hline Mean age (SD) & $51.3(16.2)$ & $40.2(11.5)$ & $47.0(15.3)$ \\
\hline People of color, $n(\%)$ & $9(56.3)$ & $10(100.0)$ & $19(73.1)$ \\
\hline \multicolumn{4}{|l|}{ Payer, n (\%) } \\
\hline Medicare & $7(43.8)$ & $4(40.0)$ & $11(42.3)$ \\
\hline Private & $2(12.5)$ & $2(20.0)$ & $4(15.4)$ \\
\hline Median number admissions in past year (IQR) & $7.0(6.0-9.5)$ & $10.0(7.0-17.0)$ & $8.5(6.0-11.0)$ \\
\hline Median number of unplanned 30-day readmissions in past year (IQR) & $4.0(3.0-4.5)$ & $7.5(3.0-11.0)$ & $4.0(3.0-8.0)$ \\
\hline
\end{tabular}

Abbreviations: IQR, interquartile range; SD, standard deviation.

prior 12 months, in addition to one or more of the following criteria: (1) at least one readmission in the last six months; (2) a referral from one of the patient's medical providers; or (3) at least three observation visits. We excluded patients whose preferred language was not English and those disoriented to person, place, or time. Considering $\mathrm{NMH}$ data showing that approximately one-third of high-utilizer patients have sickle cell disease, we used purposive sampling with the goal to compare findings within and between two groups of participants; those with and those without sickle cell disease. Our study was deemed exempt by the Northwestern University Institutional Review Board.

\section{Participant Enrollment and Data Collection}

We created an interview guide based on the research team's experience with this population, a literature review, and our research question (See Appendix). ${ }^{8,9}$ A research coordinator approached eligible participants during their hospital stay. The coordinator explained the study to eligible participants and obtained verbal consent for participation. The research coordinator then conducted one-on-one semi-structured interviews. Interviews were audio recorded for subsequent transcription and coding. Each interview lasted approximately 45 minutes. Participants were compensated with a $\$ 20$ gift card for their time.

\section{Analysis}

Digital audio recordings from interviews were transcribed verbatim, deidentified, and analyzed using an iterative inductive team-based approach to coding. ${ }^{10}$ In our first cycle coding, all coders (KJO, SF, MMC, LO, KAC) independently reviewed and coded three transcripts using descriptive coding and subcoding to generate a preliminary codebook with code definitions. ${ }^{10,11}$ Following the meetings to compare and compile our initial coding, each researcher then independently recoded the three transcripts with the developed codebook. The researchers met again to triangulate perspectives and reach a consensus on the final codebook. Using multiple coders is a standard process to control for subjective bias that one coder could bring to the coding process. ${ }^{12}$ Following this meeting, the coders split into two teams of two (KJO, SF, and MMC, $\mathrm{LO})$ to complete the coding of the remaining transcripts. Each team member independently coded the assigned transcripts and reconciled their codes with their counterpart; any discrepancies were resolved through discussion. Using this strategy, every transcript was coded by at least two team members. Our second coding cycle utilized pattern coding and involved identifying consistency both within and between transcripts; discovering associations between codes. ${ }^{10,11,13}$ Constant comparison was used to compare responses among all participants, as well as between sickle-cell and nonsickle-cell participants. ${ }^{13,14}$ Following team coding and reconciling, the analyses were presented to a broader research team for additional feedback and critique. All analyses were conducted using Dedoose version 8.0.35 (Los Angeles, California). Participant recruitment, interviews, and analysis of the transcripts continued until no new codes emerged and thematic saturation was achieved.

\section{RESULTS}

\section{Participant Characteristics}

Overall, we invited 34 patients to be interviewed; 26 consented and completed interviews (76.5\%). Six (17.6\%) patients declined participation, one (2.9\%) was unable to complete the interview before hospital discharge, and one (2.9\%) was excluded due to disorientation. Demographic characteristics of the 26 participants are shown in Table 1.

Four main themes emerged from our analysis. Table 2 summarizes these themes, subthemes, and provides representative quotes.

\section{Major Medical Problem(s) are Universal,} but High Hospital Use Varies in Onset

Not surprisingly, all participants described having at least one major medical problem. Some participants, such as those with genetic disorders, had experienced periods of high hospital use throughout their entire lifetime, while other participants experienced an onset of high hospital use as an adult after 


\section{TABLE 2. Themes, Subthemes, and Representative Quotes}

Theme Subtheme Representative Quotes

Major medical problems are universal, Congenital disorder but high hospital use varies in onset

I was born with sickle cell anemia... and I remember those years of going to the hospital [and] to the emergency room as a four-year-old, five-year-old, six-year-old. (Participant 26, a middle-aged male with sickle cell disease) I was born sick and I have a chronic medical condition, so I knew at a young age that this was going to be a lifelong challenge. (Participant 23, a young adult male with a rare genetic disorder)

Medical disorder diagnosed after being previously healthy
I was extremely energetic. Kayaking, gymnastics...very active lifestyle. It's gone from a 10 to a zero. Well, maybe a 1 due to the breathing. (Participant 9 , an older adult male with severe asthma and bronchiectasis)

My health was good until I turned the age of $39 \ldots$ when I found that I was diabetic. Prior to that, I was never sick. (Participant 25, a middle-aged female with diabetes mellitus, gastroparesis, and spinal abscess)
Combination of a major medical illness and other medical problems and/or complications
I have myasthenia gravis. I've had it since I was 25 , but it didn't require hospitalizations until I was about $40 \ldots$. I was in a very bad bus accident. I broke a bunch of bones. I fell on a piece of metal and it pierced my colon and introduced a super drug resistant bacteria... I've just lived in the hospital and lacute rehabilitation facilityl and in a nursing home and just bouncing back and forth. (Participant 3, a middle aged female with myasthenia gravis, morbid obesity, and complications of prior colonic perforation)

Well, right after I had my injury, that was the start. That was 1982 and since then I have had to be admitted with urinary tract infections. Like now, it is dealing with the bowel program and my bladder because I had bladder surgery and ended up having a bowel obstruction and that is what has got me mostly admitted to the hospital now. (Participant 4, an older adult male with cervical spinal cord injury and autonomic dysfunction)

Course fluctuates over time and is related to psychological, social, and illness economic factors
Psychological stress exacerbates

In my adult years, I [have had] a lot of stress. You know, stress brings a lot of pain on, too... I had an uncle just passed about a year and a half ago and my son... he wind up getting into some trouble at school. (Participant 18, a middle aged male with sickle cell disease)

I think stress has a lot to play in it. I think I have a tendency to breathe heavier and exhale with more difficulty. (Participant 9, an older adult male with severe asthma and bronchiectasis)

Social support helpful, but sometimes missing. When present, support can be bidirectional

It is good to have family to help deal with it. That helps a lot. And having family can keep you mentally strong to deal with it. (Participant 4 , an older adult male with cervical spinal cord injury and autonomic dysfunction)

I got to try to figure this out because I don't have people to talk to like other people do. I don't have friends to talk to and tell them your problems and they help you out and stuff. I don't have that. (Participant 10, an older adult male with severe COPD and morbid obesity)

My mom's boyfriend, his niece has sickle cell. She's just about to turn 13 and right before I came to the hospital, she was feeling down... and me and her, we sat down and talked and I told her "Just because you feel down now, it doesn't mean that you're going to be like this forever. And never like look at yourself as different, because everybody's different in their own way." (Participant 14, a young adult female with sickle cell disease)

Financial barriers impede care and support

I've talked to them [alcohol treatment programs]. They explain how much it is. Honestly, not everybody can afford to pay $\$ 30,000$ to go to rehab. (Participant 11, a middle aged male with alcohol abuse and acute on chronic pancreatitis) I don't have no money to get them [medications]... I use it up and they won't cover it because I have to wait thirty days for them to pay for the medicine again. I end up having to pay out of pocket because I am going four or five days without any pain relief. (Participant 2, an adult male with sarcoidosis) uncontrollable
Onset and progression of episodes vary, but generally seem

Sudden, unpredictable, and/or related It comes out of nowhere. I was just in the hospital last week for the same thing and they sent me home. But then, to factors beyond control a couple of days later, I was feeling awful. I was taking my medicine they gave me to take and it wasn't working. (Participant 1, a young adult female with diabetes mellitus and gastroparesis)

They [symptoms] come unexpectedly, like out of the blue. That morning, for example, I wake up. I take a shower, I have my coffee and breakfast. And suddenly boom. In 23 minutes I was here. (Participant 13, an older adult female with recurrent idiopathic vasodilatory/distributive shock

I will be in and out of the hospital because of the weather, the rain, or if I have a cold. It triggers it off. Being dehydrated, that's the same thing. (Participant 17, a middle aged female with sickle cell disease)

It could be food. It could be anything. I could eat the same food for a week straight, be fine, and then one day just throw it up. (Participant 22, an adult male with diabetes mellitus and gastroparesis)

High symptom burden, especially pain, Symptoms? Just everything you can think of. I have everything. (Participant 24, a middle aged male with end stage renal which interferes with daily activities disease and heart failure)

I started having a lot of throwing up and diarrhea and oh man, so much pain. (Participant 15, a middle aged male with sickle cell disease)

When I am in pain, I can't go anywhere. I just can't go anywhere when I am in pain. (Participant 4, an older adult male with cervical spinal cord injury and autonomic dysfunction)

It was hard. It got to the point where I was trying to work and I couldn't work because it was hurting me so bad and I [was] trying to use the sewing machine and it was a mess. (Participant 8, a middle aged female with sarcoidosis, complications of gastric bypass surgery, and chronic back pain)
Individuals prefer to avoid Care is sought after failure hospitalization and seek care only of self-management
I try to deal with it at home, much as I can before I come to the emergency room. If I can't deal with it at home before | wind up taking all my meds, I just have to come in. (Participant 18, a middle aged male with sickle cell disease) Sometimes I lay around the house for two or three days, just taking pain pills. And after that my sister will tell me "no, you have to got to go [to the hospitall." (Participant 7, an older adult female with history of a stroke, COPD, and complications of prior gastrointestinal surgery)

I take ondansetron for the nausea and vomiting, but when it's not controlled and I can't control it myself, I come in to Northwestern and find that I'm dehydrated. (Participant 25, a middle aged female with diabetes mellitus, gastroparesis and spinal abscess)
Patients wish to avoid hospitalization I dread coming here. (Participant 23, a young adult male with a rare genetic disorder) And Easter weekend I had just got sick that Saturday with fever... and I'm like "oh, what's wrong with me, Jesus?" because I'm in a lot of pain right here. And I went "Oh Lord, I just came home. I hope I don't have to go back to the hospital." Sure enough, I did. (Participant 17, a middle aged female with sickle cell disease)

I just want to have [it] like I used to. Where I don't have all these hospital stays and everything... I have been in the hospital a whole lot and I miss my family. (Participant 8, a middle aged female with sarcoidosis, complications of gastric bypass surgery, and chronic back pain) 
being previously healthy. Though most participants with genetic disorders had sickle cell anemia; one had a rare genetic disorder which caused chronic gastrointestinal symptoms. Participants typically described having a significant medical condition as well as other medical problems or complications from past surgery. Some participants described having a major medical problem which did not require frequent hospitalization until a complication or other medical problem arose, suggesting these new issues pushed them over a threshold beyond which self-management at home was less successful.

\section{Course Fluctuates over Time and is Related} to Psychological, Social, and Economic Factors Participants identified psychological stress, social support, and financial constraints as factors which influence the course of their illness over time. Deaths in the family, breakups, and concerns about other family members were mentioned as specific forms of psychological stress and directly linked by participants to worsening of symptoms. Social support was present for most, but not all, participants, with no appreciable difference based on whether the participant had sickle cell disease. Social support was generally perceived as helpful, and several participants indicated a benefit to their own health when providing social support to others. Financial pressures also served as stressors and often impeded care due to lack of access to medications, other treatments, and housing.

\section{Onset and Progression of Episodes Vary, but Generally Seem Uncontrollable}

Regarding the onset of illness episodes, some participants described the sudden, unpredictable onset of symptoms, others described a more gradual onset which allowed them to attempt self-management. Regardless of the timing, episodes of illness were often perceived as spontaneous or triggered by factors outside of the participant's control. Several participants, especially those with sickle cell disease, mentioned a relationship between their symptoms and the weather. Participants also noted the inconsistency in factors which may trigger an episode (ie, sometimes the factor exacerbated symptoms, while other times it did not). Participants also described having a high symptom burden with significant limitations in activities of daily living during episodes of illness. Pain was a very common component of symptoms regardless of whether or not the participant had sickle cell disease.

\section{Individuals Seek Care after Self-Management Fails and Prefer to Avoid Hospitalization}

Participants tried to control their symptoms with medications and typically sought care only when it was clear that this approach was not working, or they ran out of medications. This finding was consistent across both groups of participants (ie, those with and those without sickle cell disease). Many participants described very strong preferences not to come to the hospital; no participant described being in the hospital as a favorable or positive experience. Some participants mentioned that they had spent major holidays in the hospital and that they missed their family. No participant had a desire to come to the hospital.

\section{DISCUSSION}

In this study of frequently hospitalized patients, we found four major themes that illuminate patient perspectives about factors that contribute to high hospital use. While some of our findings corroborate those of previous studies, other emerging patterns were novel. Herein, we summarize key findings, provide context, and describe implications for the design of models of care for frequently hospitalized patients.

Similar to the findings of previous quantitative research, participants in our study described having a significant medical condition and typically had multiple medical conditions or complications. ${ }^{4-6}$ Importantly, some participants described having a major medical problem which did not require frequent hospitalization until another medical problem or complication arose. This finding suggests that there may be an opportunity to identify patients with significant medical problems who are at elevated risk before the onset of high hospital use. Early identification of these high-risk patients could allow for the provision of additional support to prevent potential complications or address other factors which may contribute to the need for frequent hospitalization.

Participants in our study directly linked psychological stress to fluctuations in their course of illness. Previous research by Mautner and colleagues queried participants about childhood experiences and early life stressors and reported that early life instabilities and traumas were prevalent among patients with high levels of emergency and hospital-based healthcare utilization. ${ }^{15}$ Our participants identified more recent traumatic events (eg, the death of a loved one and breakups) when reflecting on factors contributing to illness exacerbations; early life trauma did not emerge as an identified contributor. Of note, unlike Mautner et al., we did not ask participants to reflect on childhood determinants of disease and illness specifically. Our findings suggest that psychological stress contributes to illness exacerbation, even for those patients without other significant psychiatric conditions (eg, depressive disorder, schizophrenia). Incorporating mental health professionals into programs for this patient population may improve health by teaching specific coping strategies, including cognitive-behavioral therapy for an acute stress disorder. . $^{16,17}$

Social support was also a factor related to illness fluctuations over time. Notably, several participants indicated a benefit to their own health when providing social support to others, suggesting a role for peer support that may be reciprocally beneficial. This approach is supported by the literature. Williams and colleagues found that patients with sickle cell anemia experienced symptom improvement with peer support; ${ }^{18}$ while Johnson and colleagues recently reported a reduction in readmissions to acute care with the use of peer support for patients with severe mental illness. ${ }^{19}$

Financial constraints impeded care for some patients and served as a barrier to accessing medications, other treatments, and housing. Similar to the findings of prior quantitative re- 
search, our frequently hospitalized patients had a high proportion of patients with Medicaid and low proportion with private insurance, suggesting low socioeconomic status. ${ }^{9,20}$ We did not formally collect data on income or economic status. Interestingly, prior qualitative studies have not identified financial constraints as a major theme, though this may be explained by differences in study populations and the overall objectives of the studies. ${ }^{15,21}$ Importantly, the overwhelming majority of programs for frequently hospitalized patients identified in a recent systematic review included social workers. ${ }^{7}$ Our findings support the need to address financial constraints and the use of social workers in models of care for frequently hospitalized patients.

Many participants in our study felt that the factors contributing to exacerbations of illness were either inconsistent in their effect or out of their control. These findings have similarities to those from a qualitative study by Liu and colleagues in which they interviewed 20 "hospital-dependent" patients over 65 years of age. ${ }^{21}$ Though not explicitly focused on factors contributing to exacerbations, participants in their study felt that hospitalizations were generally inevitable. In our study, participants with sickle cell disease often identified changes in the weather as contributing to illness exacerbations. The relationship between weather and sickle cell disease remains incompletely understood, with an inconsistent association found in prior studies. ${ }^{22}$

Participants in our study strongly desired to avoid hospitalization and typically sought hospital care when symptoms could not be controlled at home. This finding is in contrast to that from the study by Liu and colleagues where they found that hospital-dependent patients over 65 years had favorable perspectives of hospitalization because they felt safer and more secure in the hospital. ${ }^{21}$ Our participants were younger than those from the study by Liu and colleagues, had a high symptom burden, and may have been more concerned about control of those symptoms than the risk for clinical deterioration. Programs should aim to strengthen their support of patients' self-management efforts early in the episode of illness and potentially offer home visits or a day hospital to avoid hospitalization. A recent systematic review found evidence that alternatives to inpatient care (eg, hospital-at-home) for low risk medical patients can achieve comparable outcomes at lower costs. ${ }^{23}$ Similarly, some health systems have implemented day hospitals to treat low risk patients with uncomplicated sickle cell pain. ${ }^{24,25}$

The heavy symptom burden experienced by participants in our study is notable. Pain was especially common. Programs may wish to partner with palliative care and addiction specialists to balance symptom relief with the simultaneous need to address comorbid substance and opioid use disorders when they are present. ${ }^{4,9}$

Our study has several limitations. First, participants were recruited from the medicine service at a single academic hospital using criteria we developed to identify frequently hospitalized patients. Populations differ across hospitals and definitions of frequently hospitalized patients vary, limiting the generalizability of our findings. Second, we excluded patients whose pre- ferred language was not English, as well as those disoriented to person, place, or time. It is possible that factors contributing to high hospital use differ for non-English speaking patients and those with cognitive deficits.

\section{CONCLUSION}

In this qualitative study, we identified factors associated with the onset and continuation of high hospital use. Emergent themes pointed to factors which influence patients' onset of high hospital use, fluctuations in their illness over time, and triggers to seek care during an illness episode. These findings represent an important contribution to the literature because they allow patients' perspectives to be incorporated into the design and adaptation of programs serving similar populations in other health systems. Programs that integrate patients' perspectives into their design are likely to be better prepared to address patients' needs and improve patient outcomes.

\section{Acknowledgments}

The authors thank the participants for their time and willingness to share their stories. The authors also thank Claire A. Knoten PhD and Erin Lambers PhD, former research team members who helped in the initial stages of the study.

Disclosures: The authors have nothing to disclose.

Funding: This project was funded by Northwestern Memorial Hospital and the Northwestern Medical Group.

\section{References}

1. Centers for Medicare \& Medicaid Services. Readmissions Reduction Program. http://www.cms.gov/Medicare/Medicare-Fee-for-Service-Payment/AcutelnpatientPPS/Readmissions-Reduction-Program.html. Accessed September 17, 2018.

2. Wasfy JH, Zigler CM, Choirat C, Wang Y, Dominici F, Yeh RW. Readmission rates after passage of the hospital readmissions reduction program: a prepost analysis. Ann Intern Med. 2016;166(5):324-331. https://doi.org/10.7326/ m16-0185.

3. Blumenthal D, Chernof B, Fulmer T, Lumpkin J, Selberg J. Caring for highneed, high-cost patients-an urgent priority. N Engl J Med. 2016;375(10):909911. https://doi.org/10.1056/nejmp1608511.

4. Szekendi MK, Williams MV, Carrier D, Hensley L, Thomas S, Cerese J. The characteristics of patients frequently admitted to academic medical centers in the United States. J Hosp Med. 2015;10(9):563-568. https://doi. org/10.1002/jhm.2375.

5. Dastidar JG, Jiang M. Characterization, categorization, and 5-year mortality of medicine high utilizer inpatients. J Palliat Care. 2018;33(3):167-174. https:// doi.org/10.1177/0825859718769095

6. Mudge AM, Kasper K, Clair A, et al. Recurrent readmissions in medical patients: a prospective study. J Hosp Med. 2010;6(2):61-67. https://doi. org/10.1002/jhm.811.

7. Goodwin A, Henschen BL, Odwyer LC, Nichols N, Oleary KJ. Interventions for frequently hospitalized patients and their effect on outcomes: a systematic review. J Hosp Med. 2018;13(12):853-859. https://doi.org/10.12788/jhm.3090.

8. Gelberg L, Andersen RM, Leake BD. The behavioral model for vulnerable populations: application to medical care use and outcomes for homeless people. Health Serv Res. 2000;34(6):1273-1302.

9. Rinehart DJ, Oronce C, Durfee MJ, et al. Identifying subgroups of adult superutilizers in an urban safety-net system using latent class analysis. Med Care. 2018;56(1):e1-e9. https://doi.org/10.1097/mlr.0000000000000628.

10. Miles MB, Huberman M, Saldana J. Qualitative Data Analysis. 3rd ed. Thousand Oaks, California: SAGE Publications; 2014.

11. Saldana J. The Coding Manual for Qualitative Researchers. Thousand Oaks, California: SAGE publications; 2013.

12. Lincoln YS, Guba EG. Naturalistic Inquiry. 1 ed. Beverly Hills, California: SAGE Publications; 1985

13. Kolb SM. Grounded theory and the constant comparative method: valid research strategies for educators. J Emerging Trends Educ Res Policy Stud. 
2012;3(1):83-86.

14. Glasser BG, Strauss AL. The Discovery of Grounded Theory: Strategies for Qualitative Research. New York: Taylor and Francis Group; 2017

15. Mautner DB, Pang H, Brenner JC, et al. Generating hypotheses about care needs of high utilizers: lessons from patient interviews. Popul Health Manag 2013;16(Suppl 1):S26-S33. https://doi.org/10.1089/pop.2013.0033.

16. Carpenter JK, Andrews LA, Witcraft SM, Powers MB, Smits JAJ, Hofmann SG Cognitive behavioral therapy for anxiety and related disorders: a meta-analysis of randomized placebo-controlled trials. Depres Anxiety. 2018;35(6):502 514. https://doi.org/10.1002/da.22728.

17. Roberts NP, Kitchiner NJ, Kenardy J, Bisson JI. Systematic review and meta-analysis of multiple-session early interventions following traumatic events. Am J Psychiatry. 2009;166(3):293-301. https://doi.org/10.1176/appi. ajp.2008.08040590.

18. Williams H, Tanabe P. Sickle cell disease: a review of nonpharmacological approaches for pain. J Pain Symptom Manag. 2016;51(2):163-177. https:// doi.org/10.1016/j.jpainsymman.2015.10.017.

19. Johnson S, Lamb D, Marston L, et al. Peer-supported self-management for people discharged from a mental health crisis team: a randomised controlled trial. Lancet. 2018;392(10145):409-418. https://doi.org/10.1016/s0140-
6736(18)31470-3

20. Mercer T, Bae J, Kipnes J, Velazquez M, Thomas S, Setji N. The highest utilizers of care: individualized care plans to coordinate care, improve healthcare service utilization, and reduce costs at an academic tertiary care center. $J$ Hosp Med. 2015;10(7):419-424. https://doi.org/10.1002/jhm.2351.

21. Liu T, Kiwak E, Tinetti ME. Perceptions of hospital-dependent patients on their needs for hospitalization. J Hosp Med. 2017;12(6):450-453. https://doi. org/10.12788/jhm.2756

22. Piel FB, Steinberg MH, Rees DC. Sickle cell disease. N Engl J Med. 2017;376(16):1561-1573. https://doi.org/10.1056/nejmra1510865.

23. Conley J, O'Brien CW, Leff BA, Bolen S, Zulman D. Alternative strategies to inpatient hospitalization for acute medical conditions: a systematic review. JAMA Intern Med. 2016;176(11):1693-1702.https://doi.org/10.1001/jamainternmed.2016.5974.

24. Adewoye AH, Nolan V, McMahon L, Ma Q, Steinberg MH. Effectiveness of a dedicated day hospital for management of acute sickle cell pain. Haematologica. 2007;92(6):854-855. https://doi.org/10.3324/haematol.10757.

25. Benjamin LJ, Swinson Gl, Nagel RL. Sickle cell anemia day hospital: an approach for the management of uncomplicated painful crises. Blood. 2000;95(4):1130-1136.

\section{Help Improve Your Patient Outcomes and Help Reduce Healthcare Costs}

Bring the Society of Critical Care Medicine's educational content to your institution or organization.

All courses combine dynamic presentations with hands-on skill stations to form a thorough learning experience and prepare healthcare professionals to manage critically ill and injured patients.

\section{Ultrasound Courses}

- Critical Care Ultrasound: Adult

- Critical Care Ultrasound: Pediatric and Neonatal

- Advanced Critical Care Ultrasound: Adult

Society of

Critical Care Medicine

\section{Fundamentals Courses}

- Fundamental Critical Care Support

- Pediatric Fundamental Critical Care Support

- Fundamental Disaster Management

- Fundamental Critical Care Support: Tropical Diseases

- Fundamental Critical Care Support: Obstetrics

Onsite staff training is the perfect way to provide consistent and uniform training to healthcare professionals across an institution or organization.

\section{Contact the Licensing Team or visit the website to learn more and start planning your course today!}

